\title{
Control Mechanism Analysis of Bone Remodelling under Mechanical Stimuli
}

\author{
YANAN WANG ${ }^{1, a^{*}, \text { HUAIWEN WANG }}{ }^{2, b}$ \\ ${ }^{1}$ School of Engineering, Deakin University, Waurn Ponds campus, Geelong, Vic 3216, Australia \\ ${ }^{2}$ School of Mechanical Engineering, Tianjin University of Commerce, Tianjin, 300134, China \\ ayanan.wang@deakin.edu.au, bwanghw@tjuc.edu.cn
}

\begin{abstract}
Keywords: bone remodelling, control mechanism, mechanical stimuli
Abstract. In this paper the control mechanism of bone remodelling under mechanical stimuli at cellular level is investigated by means of an extensive parametric study on a theoretical model developed previously. From a perspective of control mechanism, it is found that there are several control mechanisms working simultaneously in bone remodelling which is a complex process. To this purpose, an extensive parametric study has been carried out for investigating model parameter space related to cell differentiation and apoptosis which can describe the fundamental cell lineage behaviors. After analyzing all the combinations of 728 permutations in six model parameters, we have identified a small number of parameter combinations that can lead to physiologically realistic responses which are similar to theoretically idealized physiological responses. The results presented in the work enhanced our understanding on mechanical bone remodelling and the identified control mechanisms can help researchers to develop combined pharmacological-mechanical therapies to treat bone loss diseases such as osteoporosis.
\end{abstract}

\section{Introduction}

Bone remodeling is a coupled process in which there is localized removal of old bone and replacement with newly formed bone. Followed an activation-resorption-formation sequence [1], this happens in basic multicellular unit (BMU) which is a mediator mechanism bridging individual cellular activity to whole bone morphology [2]. Two principle cell types, the osteoclast cell and osteoblast cell are found in bone, which are the main effectors in bone turnover. The osteoblast cell produces the matrix which is mineralized in a well regulated manner. The mineralized bone matrix can be removed by activated osteoclast cell. This process is complicated, requiring interaction among different cell types that are regulated by a variety of biochemical and mechanical factors. Mechanical loading is a particularly potent stimulus for bone cells, which improves bone strength and inhibits bone loss with age.

The major reason for bone remodeling is to respond and adapt to the mechanical stresses which happen as a result of mechanical loading during physical exercises. Disorder in bone remodeling is common in many bone diseases such as osteoporosis and osteoarthritis [3]. The control mechanisms responsible for the dysfunction remain unclear.

Current understanding of mechanical bone remodeling is primarily based on experimental results in vivo and in vitro. A recent report[4] shows that osteocytes are the professional mechanosensory cells of bone and the lacuno-canalicular porosity is the structure that mediates mechanosensing. It is also shown that the dynamic mechanical load causes fluid flow in the lacuno-canalicular network [5]. Wang and Qin [6] proposed a bone cell population dynamics model for cortical bone remodeling under mechanical stimulus. However, no control mechanism research of mechanical bone remodeling at cellular level has been done.

In this paper we investigated the underlying control mechanisms of mechanical bone remodelling system through parametric study of the theoretical model in [32]. Six fundamental differentiation and apoptosis rate parameters of the model are combined randomly with each being up and down regulated, applied as a system perturbation to the bone remodelling system. The BMC and BFE are defined as the 
objective criteria for assessment of each parameter combination. By using Matlab to carry out the large amount of calculations we manage to obtain 728 graphs of BMC and BFE vs. model parameter combination variation as system output. After analysing all the graphs, two subsets of results are summarized.

\section{Mathematical model development}

Follow the same way in [6] here we use abbreviation form for the factors involved, such as OBU for uncommitted osteoblastic progenitors, OBP for preosteoblast, OBA for mature osteoblast, OCP for osteoclast precursor, OCA for active osteoclasts, OST for osteocytes, and we use RL for RANKL, RK for RANK, T $\beta$ for TGF- $\beta$, P2 for $\mathrm{PGE}_{2}, \mathrm{OPG}, \mathrm{NO}$ and PTH unchanged.

\section{Parametric study of control mechanism}

For normal adults, there is a balance between the amount of bone resorbed by osteoclasts and the amount of bone formed by osteoblasts [1]. In this complex process, bone is remodelled by groups of cells derived from different sources, which are usually called the basic multicellular units (BMUs) [7] that follow an activation-resorption-formation sequence event. The BMU is a mediator mechanism bridging individual cellular activity to whole bone morphology [8], which is sensitive to any changes in the bone cell microenvironment. As a result, it is expected that any modification to the component of BMU will have significant effect on its output behaviour. In this paper we are going to apply perturbations to the mechanical bone remodelling system which is in steady state by down and up regulating its six differentiation and apoptosis rate parameters $D F_{o b u}, D F_{o c p}, D F_{o b p}, A_{o b a}, A_{o c a}$ and $A_{o s t}$. In this case, we have six different parameters and each parameter could be up or down regulated, by using simple combination theory, we can calculate the number of permutation is $728=\sum_{i=1}^{6} C_{6}^{i} \cdot 2^{i}$.

Then in order to investigate the system behaviour for a wide range of changes, we now apply exponentially changed factor which is $1.5^{e x}$ to each of the six differentiation and apoptosis rate parameters, where the exponent exranges from -10 to 10 in step increase of 0.5 . The assessment of each of the parameter combination to the system behaviour is chosen as the responses of BMC and BFE which are sampled on $100^{\text {th }}$ day to stand for the maximum change. By using Matlab we can plot all the 728 graphs, then summarizing all the plots of BMC and BFE vs. variation of exponent $e x$, we find that there are three subsets of curves which are plotted in Fig.1.

Fig. 1a and Fig. 1b show an exponential increase and decrease of BMC and BFE respectively, for increasing the model parameter exponentially (exponent ex from-10 to 10). This type of behaviour is considered as physiological unrealistic from a biological viewpoint and obtained for a quite large range of model parameter combinations. On the other hand, Fig.1c represents the other extreme case where only minor changes of BMC and BFE happen during the entire range of parameter variation. These three types of response curves are excluded from our further analysis on the grounds that they do not provide an effective control mechanism for $\mathrm{BMC}$ and $\mathrm{BFE}$.

It is noticed that in a bone remodelling system without consideration of mechanical stimulus, the response involving three parameters (that is $D_{O B U} / D_{O B P} / A_{O C A}=+/-/+$ ) coincides with the known physiological action of TGF- $\beta$ on bone cells that TGF- $\beta$ promotes differentiation of osteoblast progenitors, inhibits differentiation of osteoblast precursor cells, while promoting of osteoclast apoptosis [9]. But in the case of mechanical bone remodelling this combination causes exponential increases for both BMC and BFE, which is similar to Fig.1a. In other words, with the introduction of mechanical stimulus the bone remodelling system becomes different and it deserves attention from biologists and other researchers as well. 

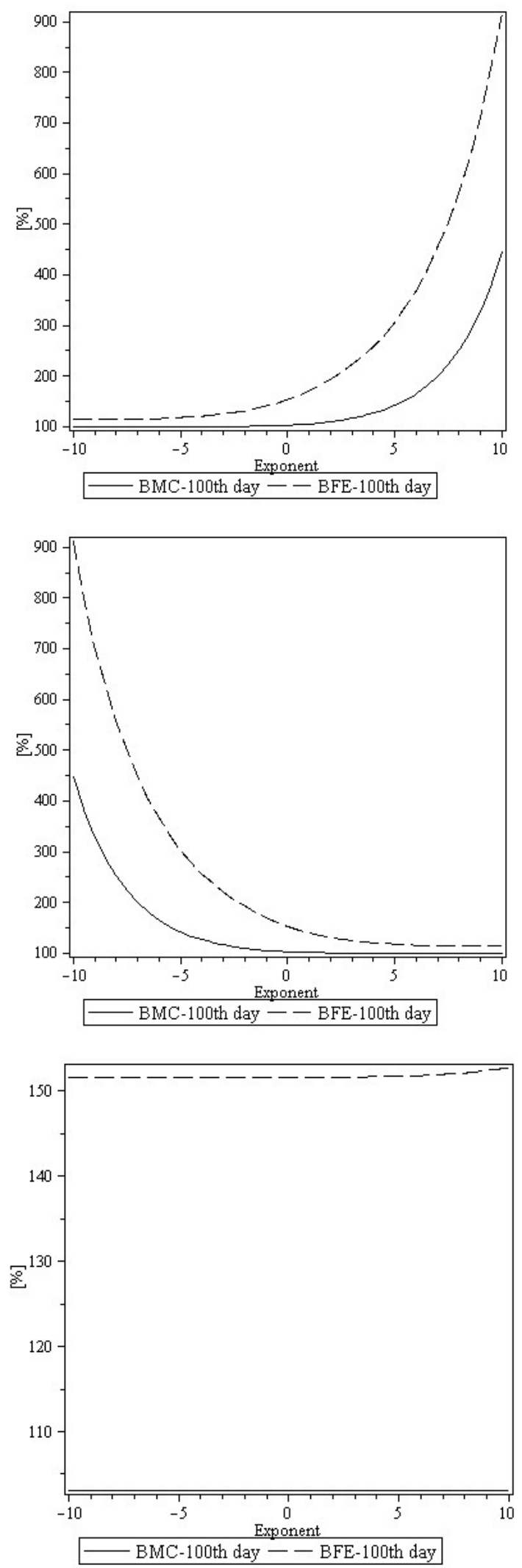

Fig. 1 Physiological unrealistic changes of BMC and BFE vs. combined changes of model parameter $\left[1.5^{-10}-1.5^{+10}\right] \cdot p:$ (a) exponential bone growth, (b) exponential bone decrease, (c) slight changes of bone. ( $p$ is parameter value). 


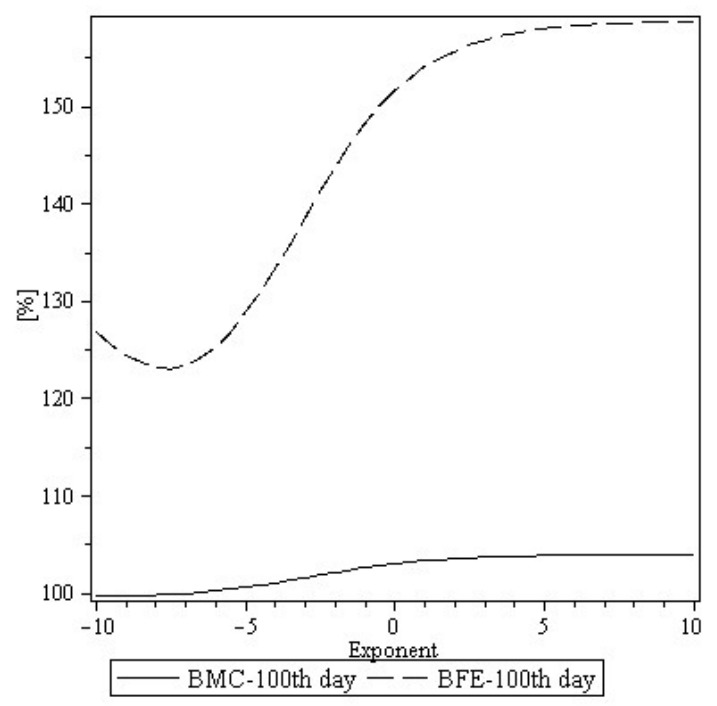

Fig. 2 Typical physiologically realistic fluctuations of BMC and BFE with combinations of parameter change.

\section{Summary and conclusion}

In this paper, based on our previous work [6], the parametric study of mechanical bone remodeling model is carried out in order to understand the control mechanism of mechanical bone remodeling at cellular level. From a control mechanism perspective, it is quite likely that there are several control mechanisms working simultaneously in bone remodeling which is a complex system. Consequently, we perform an extensive parametric study investigating model parameter space related to cell differentiation and apoptosis which describes the fundamental cell lineage behaviors, to investigate such a scenario. After analyzing all the combinations (that is 728 permutations) of six model parameters, we successfully identified a small number of parameter combinations that are able to cause physiologically realistic responses which are similar to theoretically idealized physiological response. In the end, this work will further our understanding on mechanical bone remodeling and the identified control mechanisms are able to help to develop combined pharmacological and mechanical therapies to treat bone loss diseases such as osteoporosis.

\section{References}

1. Robling, A.G., A.B. Castillo, and C.H. Turner, Biomechanical and molecular regulation of bone remodeling. Annual Reviews, 2006. 8(1): p. 455-498.

2. Frost, H.M., Intermediary organization of the skeleton. 1986: Boca Raton, Fla. : CRC Press.

3. Moroz, A., et al., Phenomenological model of bone remodeling cycle containing osteocyte regulation loop. Biosystems, 2006. 84(3): p. 183-190.

4. Nijweide, P., E. Burger, and J. Klein-Nulend, The osteocyte. Principles of bone biology, ed. John P. Bilezikian, Lawrence G. Raisz, and G.A. Rodan. 2002, San Diego CA USA: Academic Press.

5. Knothe Tate, et al., In vivo demonstration of load-induced fluid flow in the rat tibia and its potential implications for processes associated with functional adaptation. The Journal of Experimental Biology, 2000. 203(18): p. 2737-2745.

6. Parfitt, A.M., Bone effects of space flight: analysis by quantum concept of bone remodelling. Acta Astronaut, 1981. 8(9-10): p. 1083-90.

7. $\quad$ Parfitt, A.M., Osteonal and hemi-osteonal remodeling: The spatial and temporal framework for signal traffic in adult human bone. Journal of Cellular Biochemistry, 1994. 55(3): p. 273-286.

8. $\quad$ Frost, H.M., Intermediary organization of the skeleton. Boca Raton, Fl: CRC Press, 1986. 
9. Pivonka, P., et al., Model structure and control of bone remodeling: A theoretical study. Bone, 2008. 43(2): p. 249-263. 